\title{
Effect of the Random Field on the Dynamics of Pulsating, Erupting, and Creeping Solitons in the Cubic-Quintic Complex Ginzburg-Landau Equation
}

\author{
Woo-Pyo Hong \\ Department of Electronics Engineering, Catholic University of Daegu, Hayang, Gyongsan, \\ Gyungbuk 712-702, South Korea \\ Reprint requests to W.-P. H.; E-mail: wphong@cu.ac.kr
}

Z. Naturforsch. 61a, 615 - 623 (2006); received November 11, 2006

\begin{abstract}
It is shown that the dynamics of pulsating, erupting, and creeping (PEC) solitons obtained from the one-dimensional cubic-quintic complex Ginzburg-Landau equation can be drastically modified in the presence of a random background field. It is found that, when the random field is applied to a pulselike initial profile, multiple soliton trains are formed for the parameters of the pulsating and erupting solitons. Furthermore, as the strength of the gain term increases, the multiple pulsating or erupting solitons transform into fixed-shape stable solitons. This may be important for a practical use such as to generate stable femtosecond pulses. For the case of creeping soliton parameters, the presence of the random field does not generate multiple solitons, however, it induces a rapidly twisting or traveling soliton with a fixed-shape, of which stability can be also controlled by the gain term. PACS numbers: 42.65.Tg, 03.40.Kf, 05.70.Ln, 47.20.Ky.
\end{abstract}

Key words: Pulsating, Erupting, and Creeping Solitons; Random Field; Fixed-Shape Soliton.

\section{Introduction}

The complex Ginzburg-Landau equation (CGLE) is one of the widely studied nonlinear equations for describing a variety of phenomena in the pattern formation [1]. The CGLE and its extension can model a wide range of dissipative physical systems, such as binary fluid convection [2], electro-convection in nematic liquid crystals [3], patterns near electrodes in gas discharges [4], and oscillatory chemical reactions [5]. The solitons in such dissipative systems are quite different from those in conservative systems; in fact they are far from equilibrium and have nontrivial internal energy flows from an external source [6]. In particular, for the cubic-quintic extension of the CGLE, which can model the physical phenomena occurring in passively mode-locked lasers [7], pulsating, erupting and creeping (PEC) solitons have been obtained [8] and experimentally verified $[9,10]$. They strongly depend on an energy supply from an external source while continuously dissipating and redistributing the soliton's energy between its parts [9]. The dissipative solitons can take different shapes and also show a variety of periodic pulsation and chaotic explosion. In particular, the dissipative solitons behave like an attractor in the sense that a wide variety of localized initial conditions converge to it [6]. As such a model, we consider the cubicquintic complex Ginzburg-Landau equation (cqCGLE) in the context of the nonlinear optics

$$
\begin{aligned}
& \mathrm{i} \psi_{z}+\frac{d}{2} \psi_{\tau \tau}+|\psi|^{2} \psi+v|\psi|^{4} \psi \\
& =\mathrm{i} \delta \psi+\mathrm{i} \beta \psi_{\tau \tau}+\mathrm{i} \varepsilon|\psi|^{2} \psi+\mathrm{i} \mu|\psi|^{4} \psi
\end{aligned}
$$

where $d, \delta, \varepsilon, \beta, \mu$ and $v$ are real constants. The physical meaning of each particular term depends on the dynamical system under consideration. For example, in the mode-locked laser system, $\psi(z, \tau)$ is the normalized amplitude, $z$ is the propagation distance or the cavity round-trip number (treated as a continuous variable), $\tau$ is the retarded time, $d$ is the group-velocity dispersion coefficient with $d= \pm 1$ depending on anomalous $(d=1)$ or normal $(d=-1)$ dispersion, $\delta$ is the linear gain $(\delta>0)$ or loss $(\delta<0)$ coefficient, $\beta$ accounts for spectral filtering or linear parabolic gain $(\beta>0)$ due to an amplifier, the term $\varepsilon$ represents the nonlinear gain (which arises, e.g., from saturable absorption), the term with $\mu$ represents, if negative, the saturation of the nonlinear gain, and the one with the coefficient $v$ corresponds, also if negative, to the saturation of the nonlinear refractive index $[6,8,9]$. It is worth noting 

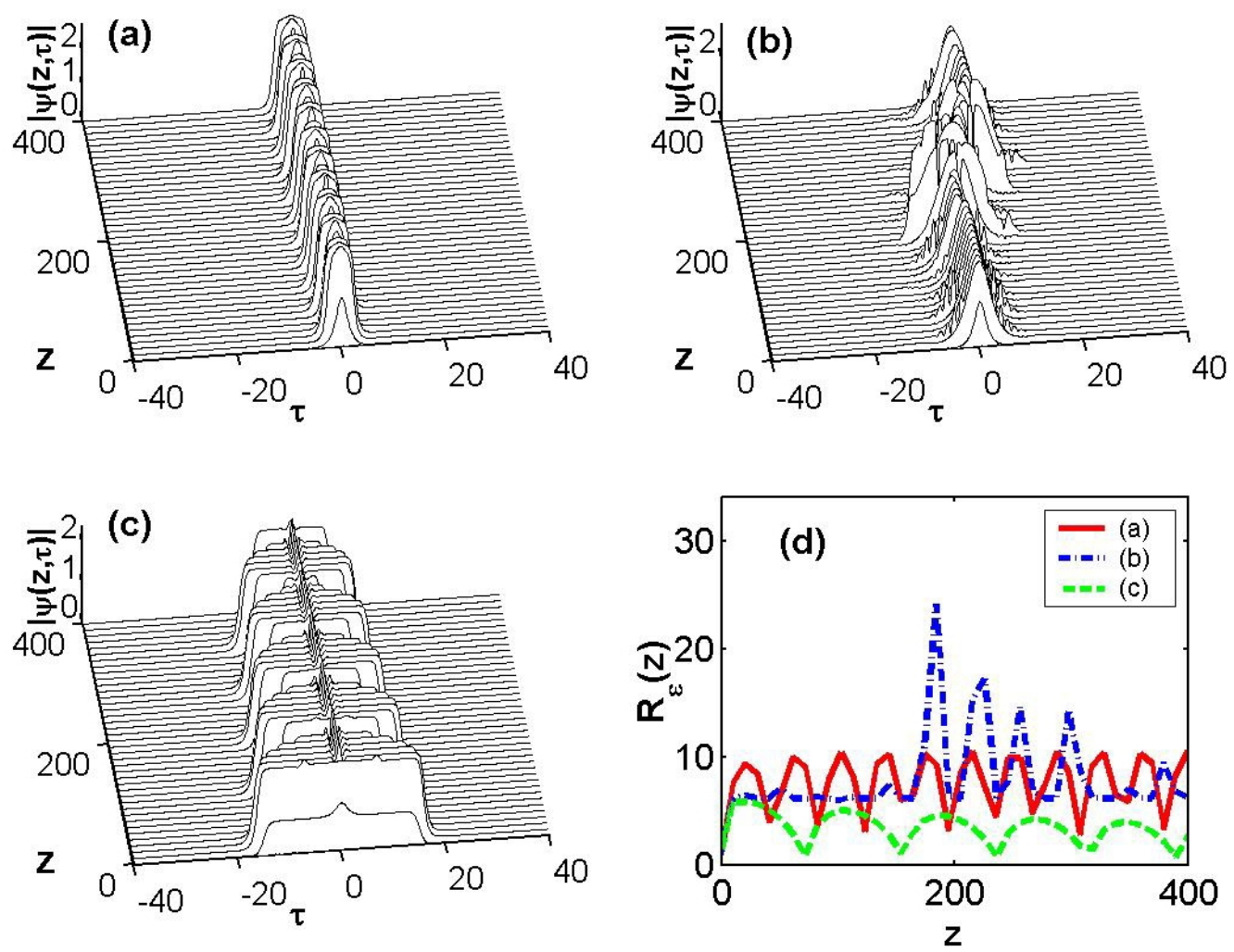

Fig. 1. Evolutions of the (a) pulsating, (b) erupting, and (c) creeping solitons, respectively, confirming the results in [8]. (d) The normalized energies $R_{\mathcal{E}}(z)$ as functions of the distance $z$ for the pulsating (solid line), erupting (dashed line), and creeping (dot-dashed line) solitons, respectively.

that for the original Ginzburg-Landau (GL) equation, which describes a large class of bifurcations and nonlinear wave phenomena in spatially extended systems, the role of the variables $z$ and $\tau$ is interchanged [10]. The reason why we name (1) as an extended GL equation is that in the laser system we are dealing with pulses which are localized entities in the time domain $\tau$ and the pulse propagates along the distance $z$.

Many interesting dynamical features beyond the dynamics of the PEC solitons have been discovered by performing numerical simulations since the large parameter ranges of the cqCGLE and its extensions are wide. For example, in the context of the cqCGLE with the parameters of the PEC solitons, Tian et al. [11] have analyzed the effect of nonlinear gradient terms on them. They have found that the nonlinear gradient terms can eliminate the periodicity of the pulsating and erupting solitons and transform them into fixedshape solitons, while for the creeping soliton, the nonlinear gradient terms can cause it to breathe periodically at different frequencies on one side and to spread rapidly on the other side. The results have shown that even small nonlinear gradient terms can cause dramatic changes in the behavior of the solution, such as causing opposite sides of the breathing solution to breathe at different frequencies. More recently, Songa et al. [12], in the context of the cqCGLE with the third-order dispersion, have shown that even small third-order dispersion can dramatically alter the behavior of the solitons such as eliminating the periodicity of the pulsating and creeping solitons, transforming them into fixedshape solitons, and altering the explosion of the erupting soliton, causing that the eruptions appear asymmetrically or the erupting soliton becomes chaotic for a little larger third-order dispersion. On the other hand, Hong [13] has previously investigated by numerical simulations the effect of the nonlinear gradient terms on the dynamics of the solitons induced by the modulational instability (MI). It was shown that small nonlinear gradient terms make the MI-induced solitons to travel along the distance and to introduce both elastic and inelastic interactions between the solitons. Very re- 
(a) $\beta=0.080$
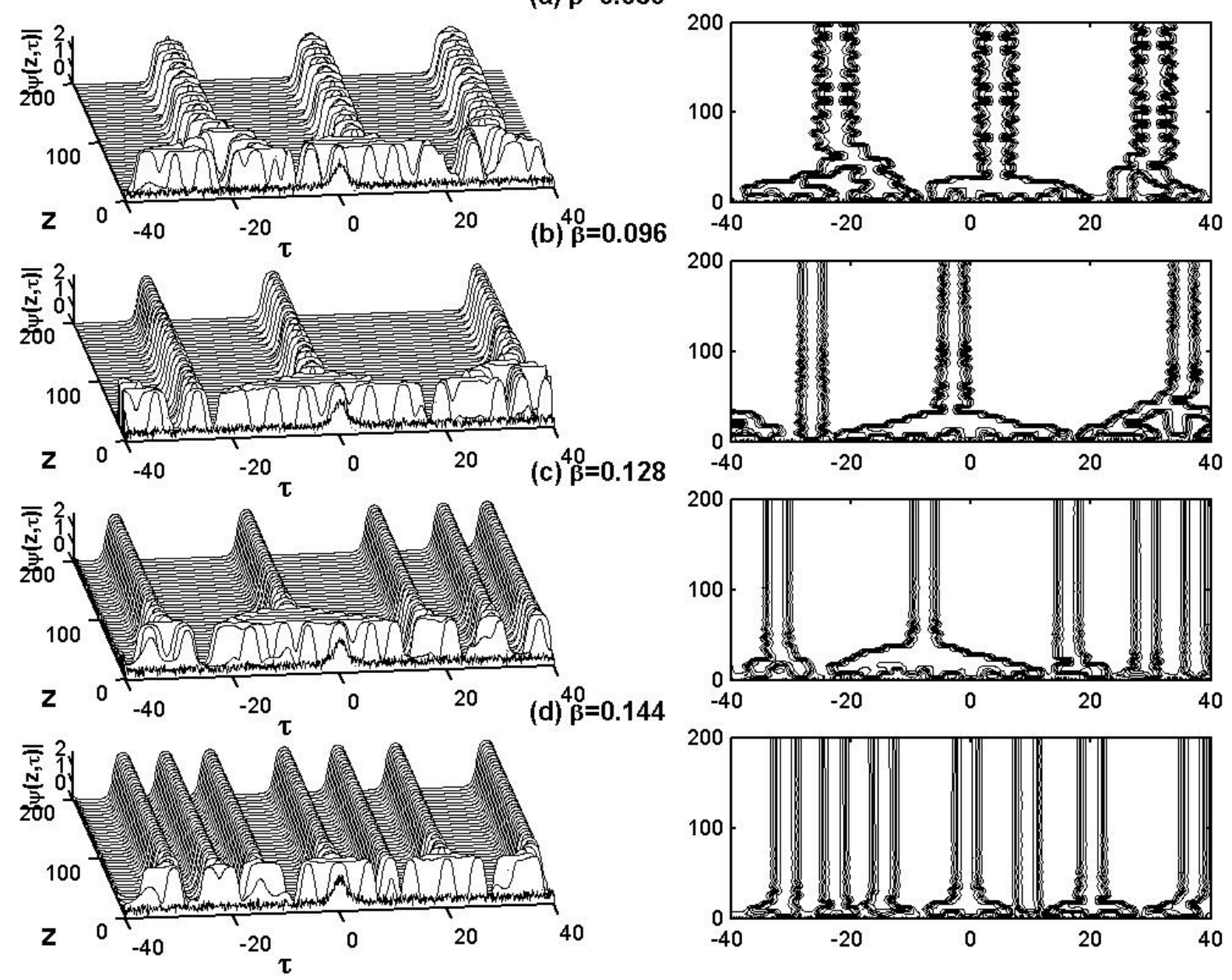

Fig. 2. Space-time evolutions and contour plots of the modulus $\psi$ for the parameters of the pulsating soliton with (a) $\beta=$ 0.080 , (b) $\beta=0.096$, (c) $\beta=0.128$, and (d) $\beta=0.144$, respectively. Multiple pulsating solitons appear in the presence of the random field in (a) and (b). Stable fixed-shape solitons are formed for the $\beta$ values in (c) and (d).

cently, Hong [14] has investigated the dynamics of the PEC solitons when the pulse-like initial profile is modulated by the MI. He has shown the formation of multiple pulsating and erupting solitons which can be transformed into the fixed-shape solitons by controlling the gain term of the cqCGLE.

The purpose of the present work is to study the effect of a random field on the dynamics of the PEC solitons in the context of the cqCGLE, similar to the recent results obtained by Hong [14]. We will show that the initial sech-type pulse in the presence of the random field produces multiple stable pulsating solitons which can evolve into stable fixed-shape solitons by controlling the spectral filtering or the linear parabolic gain value $\beta$ due to an amplifier in (1). This may be very useful in generating stable multiple femtosecond pulses in optical fibers. We will also show that the random field induces multiple erupting solitons and alters the dynamics of the solitons by mutual interaction. In addition, we will present the random field effect on the nature of the creeping soliton, of which the motion and stability can also be controlled by the gain term $\beta$.

The paper is organized as follows. In Section 2 we numerically investigate the evolutions of the pulse-type initial profile in the presence of the random field for the parameters of the PEC solitons by utilizing the splitstep Fourier method and taking the Crank-Nicholson implicit scheme for time propagation under periodic 


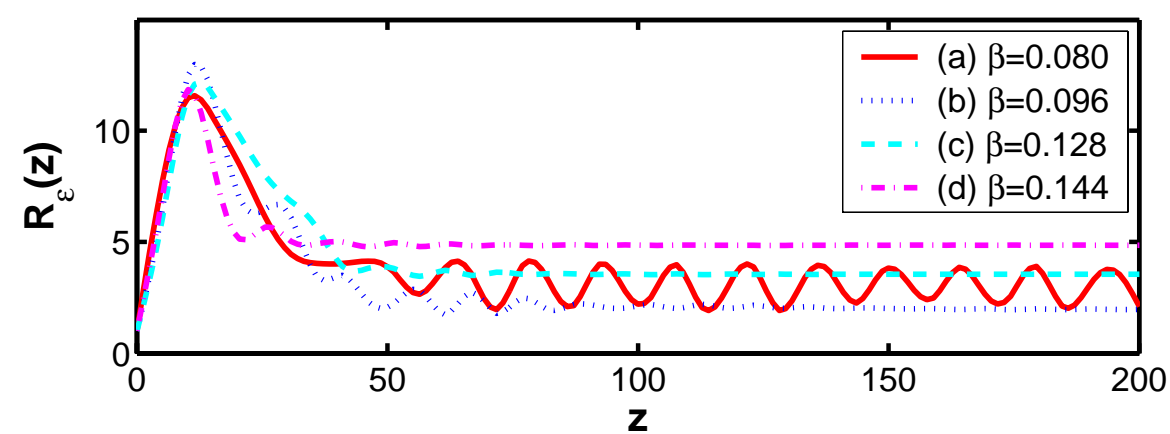

Fig. 3. Plots of the normalized energy $R_{\varepsilon}(z)$ as functions of the distance $z$ corresponding to Figs. $2 \mathrm{a}-\mathrm{d}$, respectively. The energy peaks occur during the transient stage. The pulsation in the energy converges to constant values as $\beta$ increases, indicating the stability of the fixed-shape solitons. boundary condition $[13,15,16]$. The effects of the gain term $\beta$ on the stability of the PEC solitons are presented. Our conclusions follow in Section 3.

\section{Numerical Simulations}

Before proceeding, for the sake of completeness, we would like to present in Figs. 1a-c the evolutions of the initial pulse $\psi_{0}(\tau)=\operatorname{sech}(\tau)$ for the pulsating and erupting solitons, respectively; the analytic function is given by $\psi_{0}(\tau)=\tanh (\tau+10)-\tanh (\tau-10)+$ $0.4 \operatorname{sech}(\tau)$ for the creeping soliton. The characteristics for the PEC solitons as previously found in [8] are confirmed. The parameters for the PEC solitons are: (i) pulsating soliton: $d=1, \varepsilon=0.66, \delta=-0.1$, $\beta=0.08, \mu=-0.1, v=-0.1$; (ii) erupting soliton: $d=1, \varepsilon=1.0, \delta=-0.1, \beta=0.125, \mu=-0.1, v=$ -0.1 ; (iii) creeping soliton: $d=1, \varepsilon=1.3, \delta=-0.1$, $\beta=0.101, \mu=-0.3, v=-0.101[8,9]$. The normalized energies as a function of distances, defined as $R_{\mathcal{E}}(z) \equiv \mathcal{E}(z) / \mathcal{E}(0)$ where $\mathcal{E}(z)=\int_{-\infty}^{+\infty}|\psi(z, \tau)|^{2} \mathrm{~d} \tau$, are displayed in Fig. 1d for the PEC solitons. The periodic oscillations of the pulsating (solid line) and erupting (dashed line) solitons and the rapid variation of the energy for the case of creeping (dot-dashed line) soliton are clearly demonstrated. It has been numerically confirmed, although not shown here, for a wide class of the initial localized profiles, that the PEC solitons have the property of attractors, i. e., so long as the initial profile remains a single pulse, the evolution of the pulse belongs to one of the PEC solitons. Thus, for the following analysis, we fix the initial profile as a sech function for the pulsating and erupting solitons without losing any generality.

In the rest of the paper, we will investigate the dynamical behavior of the random field if a random field is added to the initial pulse $\psi_{0}(\tau)$ in the fom

$$
\psi(0, \tau)_{\text {init }}=\psi_{0}(\tau)+\psi(\tau)_{\text {rand }}
$$

where $\psi(\tau)$ rand is the random field which may originate from a system noise or an artificially generated signal. To the author's knowledge, the space-time evolution of such initial profile for the PEC parameters has not been studied in previous works $[8,9,17]$.

\subsection{Effect of the Random Field on the Pulsating Soliton}

For the numerical computations, in order to create the random field $\psi_{\text {rand }}=\varepsilon$, where $\varepsilon$ is a random number, we choose $0 \leq \varepsilon \leq 1.0$. Then, we add the value to the initial pulse at each computational grid point in the range $|\tau| \leq 40$ to generate the initial perturbed field $\psi_{\text {init }}(\tau)$. Figure 2 shows the space-time evolutions of the modulus $\psi$ and its contour plots for the pulsating soliton parameters with varying gain $\beta$ values.

The presence of the random field results in dramatic effects on the dynamics of the pulsating soliton as shown in Fig. 2a: First, we observe the appearance of two additional pulsating solitons on the leftand the right-hand sides of the initial profile. Their behavior corresponds to the single pulsating soliton in Figure 1a. Second, we find multiple transient solitons which undergo inelastic interaction to form stable pulsating solitons, indicating the role of the random field as the seed for the solitons. Even for a slight increase in $\beta$, the pulsation of the soliton decreases, as is clearly demonstrated in the contour plot of Figure 2b. However, as $\beta$ increases up to $\beta=0.128$ in Fig. 2c, we find the transformation of the initial profile into five fixedshape solitons after a short transient distance. On the other hand, as shown in the evolution and contour plots of Fig. 2d, there appear six fixed-shape solitons showing remarkable stability with shorter transient distance. After many simulations with increasing gain value up to $\beta=0.5$, we find that the stability of the fixed-shape solitons is still maintained. 


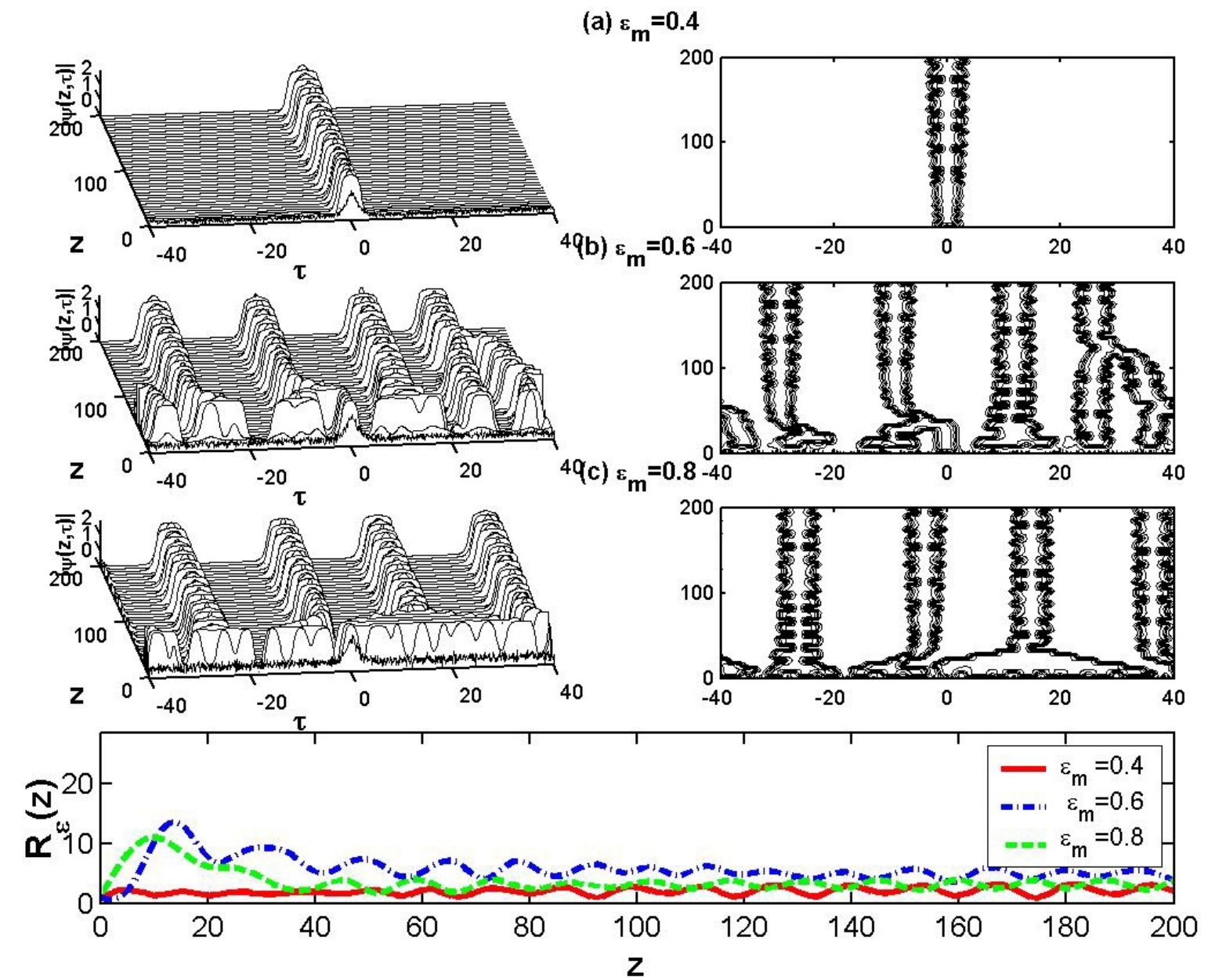

Fig. 4. Effects of varying the random field strength $\varepsilon_{\mathrm{m}}$ on the evolution of the initial pulse for (a) $\varepsilon_{\mathrm{m}}=0.4$, (b) $\varepsilon_{\mathrm{m}}=0.6$, and (c) $\varepsilon_{\mathrm{m}}=0.8$. Only for $\varepsilon_{\mathrm{m}} \geq 0.5$ the multiple pulsating solitons appear. (d) The normalized energy as function of the distance $z$. The transient energy peaks occur at an earlier stage as $\varepsilon_{m}$ increases.

In Fig. 3, we further elaborate the dynamical behavior of the solitons by calculating the evolutions of the normalized energies corresponding to Figs. $2 \mathrm{a}-\mathrm{d}$, respectively, as functions of the distance $z$. The solid line clearly demonstrates the periodic pulsations in the energy after a sharp initial peak due to the inelastic collisions between the transient solitons. Even for a slight increase in $\beta$, the energy oscillation represented by the dotted line converges to a constant value, indicating the stability of the multiple pulsating solitons. As $\beta$ increases further, both the dashed and dot-dashed lines also show flat variation in their energies after a more violent initial transient stage, during which the energy peak grows higher than that for smaller $\beta$.

To understand the relation between the maximum random field strength $\varepsilon_{\mathrm{m}}$ and the formation of the mul- tiple solitons, we simulate in Figs. $4 \mathrm{a}-\mathrm{c}$ for different $\varepsilon_{\mathrm{m}}$ with the same pulsating parameters like in Figure 1a. Figure $4 \mathrm{a}$ shows that for $\varepsilon_{\mathrm{m}}=0.4$, i. e., the random number is chosen from $0 \leq \varepsilon \leq \varepsilon_{\mathrm{m}}$, the initial profile transforms into the pulsating soliton after a short transient distance, beyond which the energy evolution has the same periodic pulsations as that in Figure 1d. We have run many simulations to find the strength of the random field required for the generation of the multiple pulsating solitons as $\varepsilon_{\mathrm{m}} \geq 0.5$. We note that, as shown in Figs. $4 \mathrm{~b}$ and $\mathrm{c}$, the number of the pulsating solitons not only depends on $\varepsilon_{\mathrm{m}}$ but also on the distribution of the random numbers. This can be noticed from the energy evolutions represented by the dashed and dot-dashed lines, respectively, showing more energy fluctuations for $\varepsilon_{\mathrm{m}}=0.8$ than that for $\varepsilon_{\mathrm{m}}=0.6$. 
(a) $\beta=0.125$
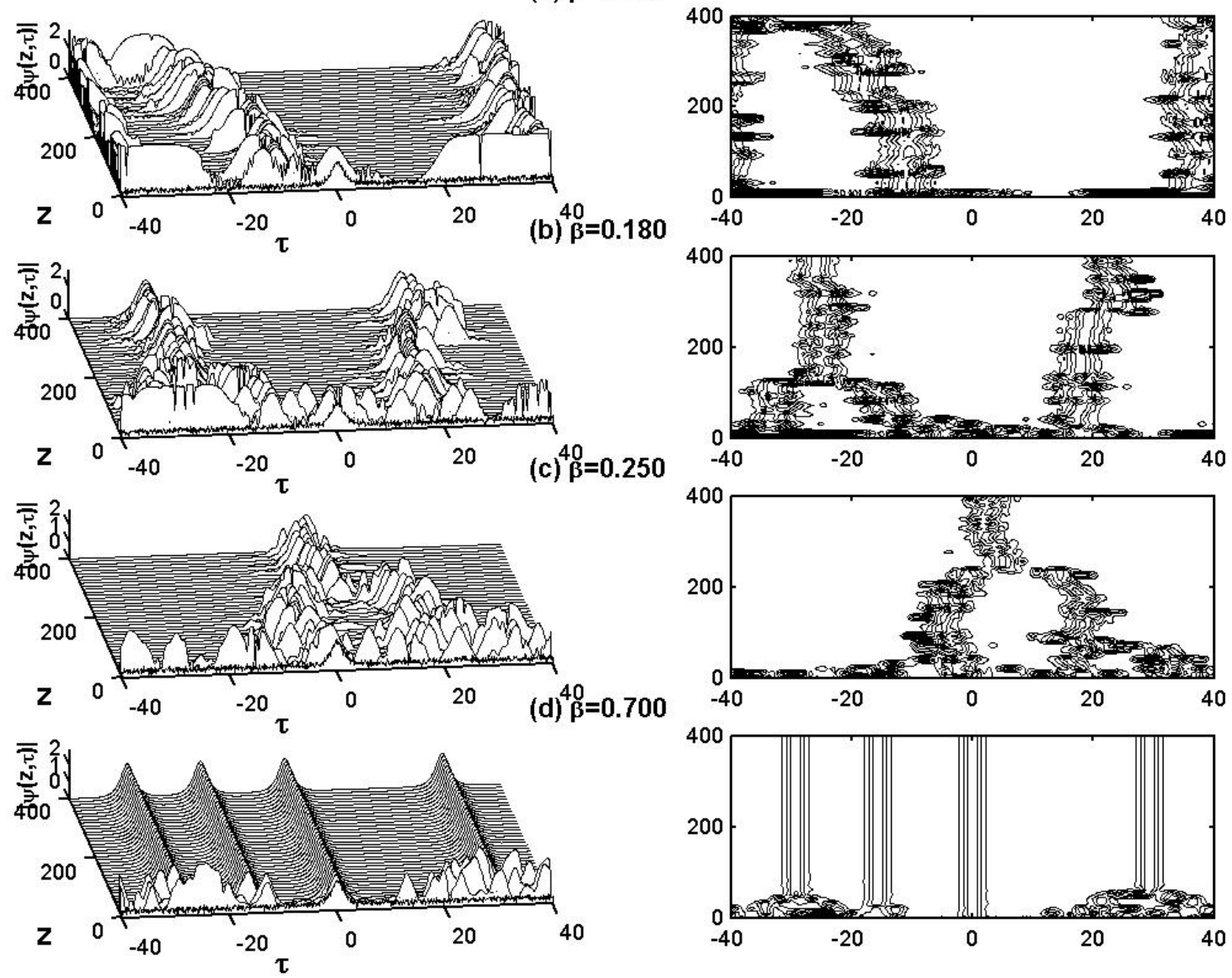

Fig. 5. Evolutions of the initial pulse with an added random field for the erupting soliton and the parameters (a) $\beta=0.125$, (b) $\beta=0.180$, (c) $\beta=0.250$, and (d) $\beta=0.700$, respectively. Two erupting solitons are shown in the presence of the random field in (a) and (b). Formation of the erupting soliton appears in (c) as a result of the inelastic collision of two transient solitons. For large $\beta$, the multiple fixed-shape solitons are formed in (d) similar to Figs. $2 \mathrm{c}-\mathrm{d}$.

In comparison with the role of the nonlinear gradient terms added to the cqCGLE, which eliminate the periodicity of the pulsating soliton and transform it into only one fixed-shape soliton, as previously discussed by Tian et al. [11], the fact that the random field generates multiple stable fixed-shape solitons by simply controlling the gain term $\beta$ is quite intriguing. It is well known that the formation of multiple solitons in mode-locked laser systems can be generated by the MI of a continuous wave [15]. However, for the parameters of PEC solitons corresponding to Figs. 1a-c, the continuous waves under MI only generate fronts as recently investigated by Hong [14]. Furthermore, even for the other parameters region allowing the existence of multiple solitons by MI, the multiple solitons can inelastically interact with each other along the propagation distance so that it may not be practical to them as the femtosecond pulses for an optical system [13]. Thus, our results can be experimentally more useful and feasible for the generation of femtosecond pulses due to simple controlling of the gain value $\beta$. Finally, we have confirmed the stability of multiple solitons up to $z=1000$ for various magnitudes of the random field.

\subsection{Effect of the Random Field on the Erupting Soliton}

Next, we investigate the effect of a random field on the evolution of the erupting soliton, which undergoes 


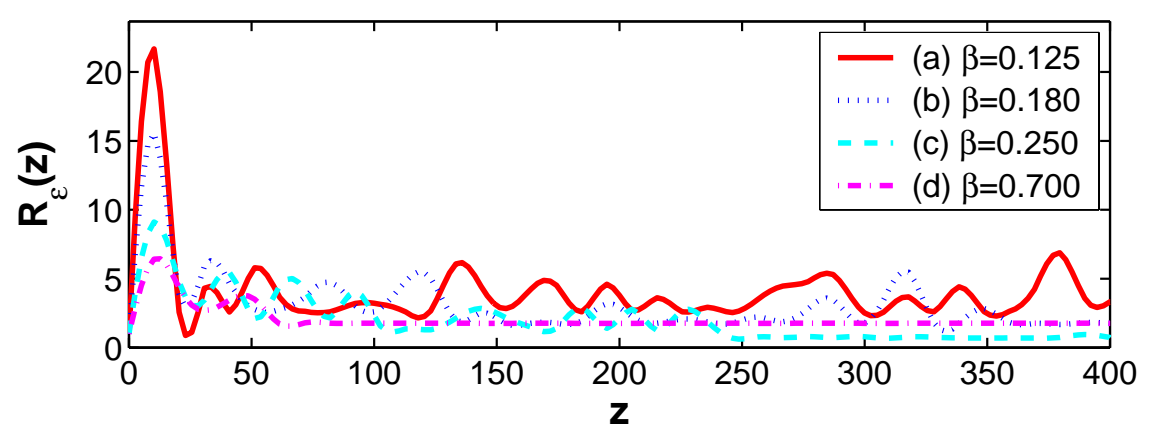

Fig. 6. Plots of the normalized energy $R_{\varepsilon}(z)$ as functions of the distance $z$ corresponding to Figs. $5 \mathrm{a}-\mathrm{d}$, respectively. The overall effect of the gain term $\beta$ on the energy evolution is to decrease the fluctuations when the strength increases. The energy peaks correspond to the distances where inelastic collision occurs between the transient solitons. an explosive transient stage before returning to its initial state $[8-10,17]$. The space-time evolutions and the contour plots of the modulus $\psi$ are shown in Fig. 5 for the erupting soliton parameters as defined in Fig. $1 \mathrm{~b}$ with random numbers chosen from $0 \leq \varepsilon \leq 1$ for the $\beta$ values.

It is shown in Fig. 5a that the random field generates two erupting solitons: the traveling soliton from the initial pulse at the center and the one from the decay of the 'front-like' structure induced by the random field. For a slightly increased $\beta$ value, we find that the initial pulse transforms into an erupting soliton which travels to the left and interacts with the transient soliton from the decay of the front-like structure at $z \approx 100$, as shown in the contour plot in Figure $5 \mathrm{~b}$. However, the erupting soliton induced from the random field propagates freely. For $\beta=0.250$ in Fig. $5 \mathrm{c}$, we observe the formation of the erupting soliton at $z \approx 250$ as a result of the inelastic collision between the soliton from the initial pulse and the other one induced from the random field. A more dramatic transformation occurs in Fig. 5d, where the strength of the gain value increases up to $\beta=0.700$. The combined effects of the random field and a large $\beta$ value let the initial profile in the center transform into the stably propagating fixed-shape soliton and generate the three stable solitons from the transient structures. From the contour plot in Fig. 5d, we find that three fixedshape solitons show the behavior of strong attractor since they are formed from the transient structures induced by the initial random field, which inelastically interacts at $z \approx 40$ and $z \approx 50$. We also note that the stable solitons are more highly peaked in comparison with the fixed-shape solitons in Figs. $2 \mathrm{c}$ and d. After many simulations with varying the strength of the random field $\varepsilon_{\mathrm{m}}$ and the gain value $\beta$, we conclude that similar stable fixed-shape solitons appear for $\varepsilon_{\mathrm{m}} \geq 0.5$ and $\beta \geq 0.6$.
The overall effect of the gain term on the energy evolutions in Fig. 6 corresponding to Figs. $5 \mathrm{a}-\mathrm{d}$, respectively, is to decrease the energy fluctuations as $\beta$ increases. The solid and dotted lines for $\beta=0.125$ and $\beta=0.180$, respectively, show the highest energy peak due to the initial transient state and the subsequent peaks due to the eruptions. However, the dashed line for $\beta=0.25$ shows the highest energy peak at $z \approx 90$, induced by the inelastic collision of the transient solitons in Figure 5b. Finally, the dot-dashed line for $\beta=$ 0.700 clearly shows more stable energy variation.

\subsection{Effect of the Random Field on the Creeping Soliton}

Here, we investigate the effect of a random field on the creeping soliton which is formed when an initial rectangular pulse with two fronts and one sink on the top pulsates back and forth relative to the sink asymmetrically at the two sides of the soliton [6]. We use the same creeping soliton parameters as given in Fig. 1c and the range of the random field defined by $0 \leq \varepsilon \leq 1.0$.

It should be pointed out that in contrast to the previous cases the random field alone does not influence the dynamics of the creeping soliton as shown in Fig. 7a, since it acts as a stronger attractor than the pulsating and the erupting solitons. However, for $\beta=0.1212$ in Fig. $7 b$, the initial profile splits into the two transient solitons which inelastically interact at $z \approx 80$ to form a creeping soliton with a reduced width. A sudden change of propagation direction with a strong twisting motion of the soliton is shown at $z \approx 120$ in Figure $7 \mathrm{c}$. For a further increased gain at $\beta=0.1616$, cf. Fig. $7 d$, the initial profile after a short transient period transforms into a traveling soliton with a breather-like behavior. Thus we expect, similar to the cases of the pulsating and erupting solitons, that for high $\beta$ values a 
(a) $\beta=0.101$
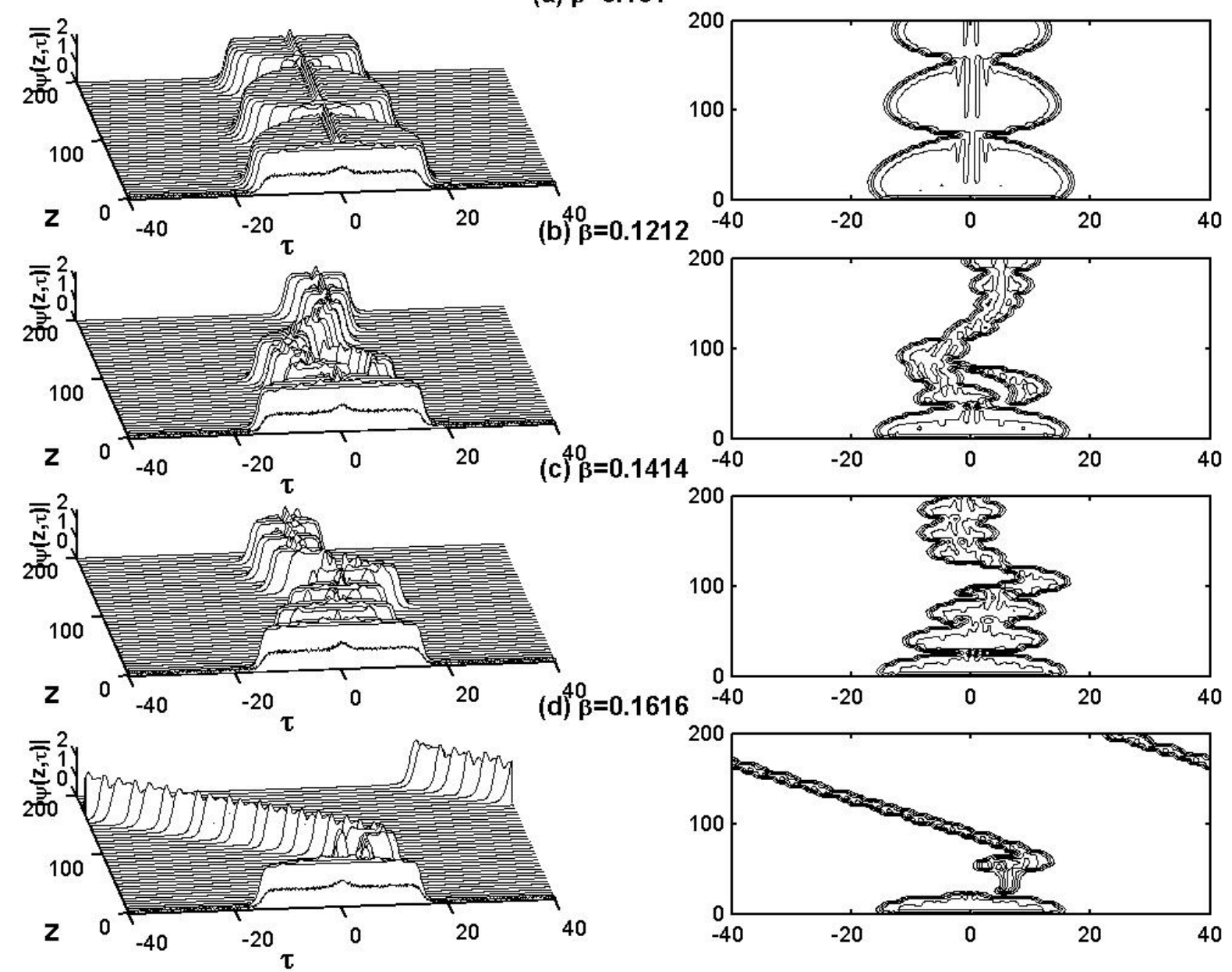

Fig. 7. Evolutions of the initial pulse including a random field for the creeping soliton parameters with (a) $\beta=0.1010$, (b) $\beta=0.1212$, (c) $\beta=0.1414$, and (d) $\beta=0.1616$, respectively. (a) A similar evolution as for the creeping soliton is shown. (b) The initial profile splits into a rapidly creeping and transient soliton, which coalesce at $z \approx 80$. (c) More twisting and sudden change of propagation direction is found. (d) The initial profile after a short transient period transforms into a traveling soliton with breathing behavior.

traveling stable soliton with fixed-shape can be generated. Finally, we note that the characteristic behaviors of the creeping solitons in Fig. 7 can only occur in the presence of both the random field and large gain.

The solid line in Fig. 8 shows a periodic pulsation in the energy evolution similar to that of the creeping soliton in Figure 1d. The dotted and dashed lines, respectively, show energy pulsations with gradually decreasing peaks as $\beta$ increases. The dot-dashed line demonstrates the stability of the soliton, which shows flat energy variation after the second peak at $z \approx 60$ where the creeping soliton transforms into the stable breathing soliton.

\section{Conclusion}

In this work, we have numerically investigated the effects of a random field and the variation of the strength of a gain term on the dynamics of the pulsating, erupting, and creeping solitons, respectively, in the context of the cubic-quintic Ginzburg-Landau equation. It has been shown that for the parameters of the pulsating and erupting solitons the sech-type initial pulse added by a random field induces multiple solitons which subsequently transform into stable fixed-shape solitons as the strength of the gain term $\beta$ increases, as shown in Figs. 2 and 5. These are very 


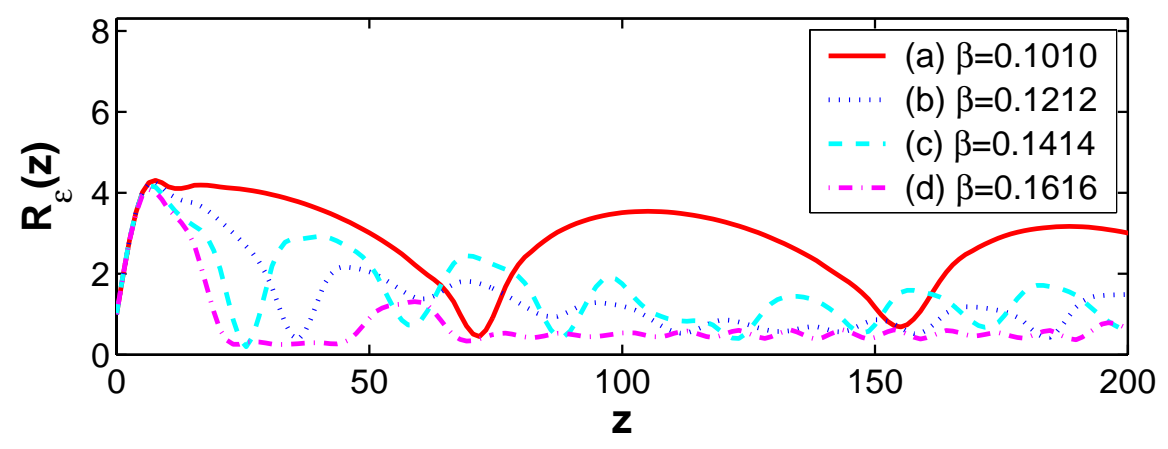

Fig. 8. Plots of the normalized energy $R_{\varepsilon}(z)$ as functions of the distance $z$ corresponding to Figs. $7 \mathrm{a}-\mathrm{d}$, respectively. The overall effect of the gain term $\beta$ on the energy evolution is to decrease the energy fluctuations when the strength increases. The stability of the breathing soliton (dot-dashed line) is demonstrated by the almost flat energy variation. similar to the formation of stable multiple solitons for the initial profile added by a modulated field [14]. The presence of the random field induces more complex transient states, after which two interacting erupting solitons are formed, as shown in Figure 5. However, for a large gain value $\beta=0.7$, we have found more highly peaked multiple solitons in comparison with those in Figs. 1c and d. Finally, we have demonstrated in Fig. 7 that the random field itself does not generate multiple solitons, in contrast to the case of pulsating and erupting solitons. However, for sufficiently large $\beta$ values, more dynamically versatile soliton motions

[1] I. S. Aranson and L. Kramer, Rev. Mod. Phys. 74, 99 (2002).

[2] P. Kolodner, Phys. Rev. A 44, 6448 (1991).

[3] M. Dennin, G. Ahlers, and D. S. Cannell, Phys. Rev. Lett. 77, 2475 (1996).

[4] K. G. Müller, Phys. Rev. A 37, 4836 (1988).

[5] Y. Kuramoto, Chemical Oscillations, Waves and Turbulence, Springer, Berlin 1984.

[6] N. Akhmediev and J. M. Soto-Crespo, Phys. Rev. E 70, 036613 (2004).

[7] H. Haus, J. Appl. Phys. 46, 3049 (1975).

[8] J. M. Soto-Crespo, N. N. Akhmediev, and A. Ankiewicz, Phys. Rev. Lett. 85, 2937 (2000).

[9] N. N. Akhmediev, J.M. Soto-Crespo, and G. Town, Phys. Rev. E 63, 056602 (2001). such as strongly creeping and twisting motions have been observed in Figs. $7 \mathrm{~b}$ and c. A traveling soliton with breather-like behavior has been found in Fig. 7d for the largest $\beta$ value.

\section{Acknowledgements}

The author are very grateful to the editor Prof. S. Grossmann for his valuable comments and thanks the anonymous referee for very detailed corrections and suggestions of the manuscript. This work was supported by the second stage of Brain Korea 21 (2006).

[10] S. T. Cundiff, J.M. Soto-Crespo, and N. Akhmediev, Phys. Rev. Lett. 88, 073903-1 (2002).

[11] H.P. Tian, Z.H. Li, J.P. Tian, G. S. Zhou, and J. Zi, Appl. Phys. B 78, 199 (2004).

[12] L. Songa, L. Lia, Z. Lia, and G. Zhoua, Opt. Comm. 249, 301 (2005).

[13] W. P. Hong, Z. Naturforsch. 61a, 23 (2006).

[14] W. P. Hong, Z. Naturforsch. 61a, 525 (2006).

[15] G. P. Agrawal, Nonlinear Fiber Optics, Optics and Photonics, 3rd ed., Academic Press, New York 2001.

[16] W. P. Hong, Z. Naturforsch. 60a, 719 (2005).

[17] J. M. Soto-Crespo, N. N. Akhmediev, and G. Town, J. Opt. Soc. B 19, 234 (2002). 